\title{
MODOS DE CONDUCIR LAS RELACIONES INTERPERSONALES EN INTERACCIONES DE ATENCIÓN AL PÚBLICO: EL CASO DE LAS FARMACIAS EN SEVILLA Y LONDRES ${ }^{1}$
}

\author{
MARIOLA HeRnáNDEZ López \\ Universidad de Greenwich \\ María Elena Placencia \\ Birkbeck College, Universidad de Londres
}

\begin{abstract}
Resumen
This paper offers a contrastive analysis of the "rapport management" strategies (cfr. SpencerOatey, 2000) employed by pharmacists and their costumers in interactions in pharmacies in Seville and London. The behaviour of Sevillians and Londoners was found to vary along five main dimensions or "socio-pragmatics interactional principles" (cfr. Spencer-Oatey and Jiang, 2003): (1) formality vs. informality, (2) consensus vs. self-affirmation, (3) dyadic vs. gregarious / public communication, (4) restraint vs. talkativeness, and (5) directness vs. indirectness in the formulation of the transation. Also following Spencer-Oatey (in press), the paper attempts to explain the motivations behind the preferences exhibited by bouth groups, with particular reference to considerations of "face", the goals that the interactants pursue, and their rights and obligations in relation to the type of activity that they are engaged in.
\end{abstract}

\section{Introducción}

En este trabajo ofrecemos un análisis contrastivo exploratorio de los modos de conducir las relaciones interpersonales de farmaceúticos y clientes en Sevilla y Londres. Es un trabajo que se enmarca en el área de la comunicación transcultural y que intenta identificar y explicar aspectos de variación en la comunicación de dos grupos socioculturales distintos.

Nos hemos apoyado en términos generales en la teoría de la gestión interrelacional $(\mathrm{GI})^{2}$ (rapport management) de Spencer-Oatey (2000; en prensa) y Spencer-Oatey y Jiang (2003) que, desde nuestro punto de vista, ofrece una alternativa más adecuada que la de Brown y Levinson (1987), la teoría todavía de uso más extendido en el mundo anglosajón ${ }^{3}$.

\footnotetext{
I Agradecemos a Carmen García sus comentarios a una versión anterior de este trabajo.

2 Hemos tomado la traducción de rapport management como "gestión interrelacional" de Fant y Granato (2002).

3 Decimos en "términos generales" puesto que la aplicación de las propuestas de Spencer-Oatey (2000; en prensa) y Spencer-Oatey y Jiang (2003) no están libres de dificultades. Por ejemplo, las distinciones sutiles que hace Spencer-Oatey (en prensa), de tipos de imágenes (ej. quality face vs. respectability face) provienen del análisis de informes de incidentes críticos (critical incidents reports) y no de interacciones y, si bien intentan responder a la naturaleza compleja de la interacción, no son de fácil aplicación al examinar datos de interacciones.
} 
Nos ha servido de base también el trabajo de House (cfr. House, 1996; 2000) y otros investigadores que se han ocupado de examinar variación transcultural en el uso de la lengua y que han identificado algunos "principios de estilo comunicativo" (Fant, 1989) o "dimensiones" (House, 1996; 2000) de variación. Spencer-Oatey y Jiang (2003) se refieren a dichas dimensiones como "principios sociopragmáticos de interacción" (PSIs) (sociopragmatic interactional principles o SIPS). Adoptando la terminología de SpencerOatey y Jiang (2003), en este estudio hemos identificado cinco SIPs centrales mediante los cuales se puede diferenciar el comportamiento de los londinenses y sevillanos. Finalmente, nos hemos apoyado también en estudios sobre el estilo comunicativo de los españoles como los de Fant (1989), Bravo (1999) y Hernández Flores (1999), fundamentados en buena medida en el trabajo de Thurén (1988), en el cual también nos hemos basado.

Siguiendo a Spencer-Oatey (en prensa) intentamos también explicar las motivaciones detrás de las preferencias identificadas que incluirían consideraciones de imagen (face), las metas que persiguen los participantes (interactional wants en Spencer-Oatey, en prensa), y los derechos y obligaciones (rights and obligations) de los participantes, asociados con la actividad. Sin embargo, antes de describir e ilustrar los PSIs (apartado 4) y motivaciones identificadas en el presente estudio (apartado 5), consideraremos brevemente el marco teórico de Spencer-Oatey y Spencer-Oatey y Jiang con respecto a modelos tradicionales de cortesía (apartado 2) y los datos empleados en el presente estudio (apartado 3).

\section{Modelos de cortesía y el modelo de la GI}

Las propuestas de Spencer-Oatey (en prensa), y Spencer-Oatey y Jiang (2003) (véase también Spencer-Oatey, 2000; 2002) constituyen un intento de responder a varias de las críticas que se han hecho a los modelos tradicionales de cortesía, principalmente el de Brown y Levinson (1987), pero también el de Leech (1983) y Fraser (1990) (véase además Fraser y Nolen, 1981). De hecho, Spencer-Oatey y Spencer-Oatey y Jiang intentan reunir aspectos esenciales de cada una de estas propuestas en un solo marco de estudio.

Para empezar, Spencer-Oatey y Spencer-Oatey y Jiang proponen examinar la cortesía dentro del estudio de la GI, dadas las dificultades que la noción de cortesía (politeness) presenta $^{4}$ y puesto que no hay consenso sobre el significado de esta noción entre estudiosos del tema. Por citar algunos ejemplos, para Brown y Levinson (1987) la cortesía se refiere al uso de estrategias encaminadas a proteger la imagen de los participantes frente a actos amenazantes en cuanto se busca evitar conflictos en la interacción. Para Fraser (1990), en cambio, el ser cortés es equivalente a sujetarse a los términos y condiciones del "contrato conversacional" que se refiere a los derechos y obligaciones que los participantes asumen para una actividad determinada, pudiendo su definición ser renegociada a medida que progresa la interacción. Meier (1995) por su lado, afirma que ser cortés es ser apropiado, y por tanto, la cortesía sólo se puede juzgar en función del contexto, expectativas de los interlocutores y posibles interpretaciones que puedan darse en un intercambio comunicativo específico.

4 Janney y Arndt (1993: 20), por ejemplo, dicen que “.... the fact that different languages contain certain words that can be translated into English as 'politeness' [...] does not necessarily mean that notions of politeness are universal, or imply that words themselves are interpreted identically by native speakers of the languages. There may well be as many different notions of politeness [...] as there are different cultures and languages where such concepts are referred to." 
Además, se ha resaltado como problemático el hecho de que en la teoría de Brown y Levinson se tomen comportamientos corteses como base de la interacción social (cfr. Eelen, 2001), enfatizándose solamente estrategias encaminadas a mantener la armonía en la interacción y no dándose cabida a la explicación de comportamientos no corteses. Esto llevó a Culpeper (1996), por ejemplo, a proponer un modelo de descortesía que sirviera de complemento al de Brown y Levinson (véase también Culpeper et alii, 2003). En cambio, la GI es una noción que da cabida tanto a comportamientos corteses como descorteses, sin dar primacía ni a unos ni a otros. Así, Spencer-Oatey (2000; en prensa) destaca que diferentes actividades pueden estar encaminadas a establecer, mantener, realzar, atacar o descuidar las relaciones interpersonales 5 . Este punto está relacionado con la crítica que se ha hecho al modelo de Brown y Levinson de que, dada su visión negativa de las relaciones humanas donde prácticamente todo acto es una amenaza a la imagen de uno de los participantes en una interacción, se ha descuidado el estudio de actividades que buscan realzar su imagen sin que haya amenazas de por medio (véase Aston, 1988; Kerbrat-Orecchioni, 1997; Carrasco Santana, 1999; Hernández Flores, 2004). Por otro lado, se ha criticado que en la teoría de Brown y Levinson se enfatiza la protección de la imagen del oyente, descuidando actividades orientadas hacia la protección o realce de la imagen del propio hablante (cfr. Chen, 2001). La teoría de la GI da cabida a consideraciones de este tipo de imagen.

Más aún, mientras en la teoría de Brown y Levinson la explicación central en el uso de estrategias de cortesía es la preocupación por proteger la imagen del interlocutor (principalmente), en la GI, consideraciones de imagen constituyen solamente una de las motivaciones en el uso de diferentes estrategias ${ }^{6}$. Otras serían las de derechos y obligaciones que tienen los participantes en una determinada actividad, que Spencer-Oatey (en prensa) renombra como behavioural responsibilities 7 y que provienen del marco teórico de Fraser (1990) del "contrato conversacional", y unas terceras, las metas que persiguen los participantes en la interacción a lo que Spencer-Oatey (en prensa) se refiere como interactional wants ${ }^{8}$. Este último punto está relacionado con propuestas que se han hecho en el estudio de la comunicación fática en actividades transaccionales, por ejemplo, donde se enfatiza la multiplicidad

Véase también el trabajo de Aston (1988), que partiendo de las propuestas de Brown y Yule (1983) discute la negociación de rapport como el establecimiento y mantenimiento de las relaciones sociales que podrian estar encaminados al establecimiento y mantenimiento de relaciones satisfactorias (comity o positive rapport) $\mathrm{u}$ hostiles y conflictivas (negative rapport). Este autor también se refiere a actividades que pueden realzar (enhance) las relaciones interpersonales. Por otro lado, la noción de rapport en el sentido de rapport positivo (Aston, 1988) se la encuentra también en el trabajo de Lakoff (1976) y Tannen (1994), entre otros.

6 Hay que resaltar también que para Spencer-Oatey la noción de imagen (face) es más compleja que la de Brown y Levinson (1987). Spencer-Oatey (en prensa), por ejemplo, distingue entre repectability face (el prestigio que una persona o grupo social reclama en una comunidad determinada de manera más o menos estable en el tiempo) o a qualities face (el valor positivo, negociable y específico que cada persona reclama para sí mismo en un intercambio comunicativo determinado).

7 La noción de behavioural responsabilities (anteriormente "derechos" y "obligaciones", en Spencer-Oatey y Jiang, 2003) está asociada a las creencias que se tienen de lo que está prescrito o proscrito en un contexto sociocultural determinado.

8 Spencer-Oatey y Jiang (2003) se refieren a estas motivaciones como principios sociopragmáticos de interacción centrales en contraste con principios secundarios relacionados con las máximas de Leech (1983), mientras que los primeros son "motivaciones" de la cortesía en Spencer-Oatey (en prensa). Hemos adoptado aquí la noción de motivaciones de Spencer-Oatey (en prensa) para los principios centrales en Spencer-Oatey y Jiang (2003), y la de "principios" para los principios secundarios de Spencer-Oatey y Jiang (2003). 
de metas que los participantes pueden perseguir en una interacción (cfr. Coupland, Coupland y Robinson, 1992).

Finalmente, en cuanto a la propuesta de Leech (1983) sobre la existencia de un principio de cortesía y máximas, si bien algunos autores la han encontrado útil en estudios transculturales (cfr. Chen, 1993), una de las críticas que se ha hecho es que hay un elemento etnocentrista en sus máximas en cuanto un polo de comportamiento tiende a ser favorecido (ej. la expresión de acuerdo sobre la expresión de desacuerdo) (cfr. Spencer-Oatey y Jiang, 2003). Spencer-Oatey y Jiang, reconceptualizan estas máximas como principios sociopragmáticos de interacción (secundarios) que "guían o influyen en la producción e interpretación de la lengua en uso" (Spencer-Oatey y Jiang, 2003: 1635) (nuestra trad.) y que constituyen continuos sin que un polo sea más favorable que otro fuera de contexto, es decir que la selección de comportamientos orientados hacia cualquier punto del continuo está sujeta a variación situacional y cultural. Además, estos principios podrían tener su manifestación no sólo en actos de habla como en la teoría de Brown y Levinson, sino en el ámbito del discurso ${ }^{9}$, por último, la relevancia de un principio u otro dependerá del contexto, es decir, no hay una lista predeterminada como la de las estrategias de Brown y Levinson ${ }^{10}$. Estos principios parecen corresponder en rasgos generales a lo que House (cfr. House, 1996; 2000) se refiere como "dimensiones de variación transcultural" (dimensions of cross-cultural difference) y que otros autores como Fant (1989) exploraran anteriormente en estudios contrastivos bajo el nombre de "principios de estilo comunicativo" o "prioridades culturales". Una diferencia está, sin embargo, en que Spencer-Oatey y Jiang proponen el estudio de estos principios o dimensiones dentro de un marco global de comportamiento, es decir, dentro del estudio de la GI.

\section{Datos empleados}

El presente estudio está basado en el análisis de 82 interacciones grabadas en audio en tres farmacias, una en Sevilla (42 interacciones) y dos en Londres (40 interacciones grabadas en Greenwich y Crossharbour, dos sectores en el sudeste de Londres). Las farmacias en los dos contextos vendían productos similares (medicinas y productos de higiene y belleza), y ofrecían una sección de productos de auto-servicio (véase Traverso, 2001) al igual que otra atendida por un farmacéutico para la compra de medicinas y otros productos. En este estudio nos concentramos en interacciones del segundo tipo, es decir interacciones entre farmacéuticos y clientes en las que necesariamente los participantes tienen que comunicarse verbalmente. Las de auto-servicio se descartaron porque requieren de poca o ninguna interacción verbal.

Las grabaciones se hicieron con una grabadora de minidisco colocada en un bolso en el mostrador de venta, con el consentimiento de los farmacéuticos. Se puso un aviso para los clientes indicándoles que se iba a grabar las interacciones con fines de investigación.

\footnotetext{
9 Críticas al hecho de que Brown y Levinson (1987) descuidan el nivel del discurso al concentrarse en actos de habla pueden encontrarse por ejemplo, en Aronson y Rundström (1989) y Mao (1994), y, con relación a estudios de cortesía en español, en Garcés Conejos (1993) y Placencia (1996), entre otros.

10 Críticas al modelo de Brown y Levinson (1987) en esta área se pueden encontrar en Blum-Kulka (1987) y Bravo (1999), entre otros.
} 
Se les invitó también a pedir más información al respecto al farmacéutico si así lo deseaban. Sin embargo, ningún cliente tomó esta invitación. Es posible que tanto clientes como farmacéuticos hayan modificado su comportamiento en cierta medida al tener conciencia de las grabaciones. No obstante, se espera que la influencia haya sido mínima puesto que interacciones del tipo examinado requieren de la concentración de los participantes o la interacción se entorpece. Como dice Malone (1997: 152) "[s]elf-monitoring may occur, but conversations demand participant attention, and hence talkers are quickly drawn in ...".

En las dos ciudades, las farmacias donde se obtuvieron las grabaciones estaban ubicadas en sectores mixtos de clase obrera y clase media. Debido a la dificultad para acceder a información personal de los participantes para determinar su pertenencia a un grupo social $u$ otro no se toma en cuenta aquí el factor clase social. Tampoco se toma en cuenta el género de los participantes ni su edad. La edad de los participantes en ambos grupos fluctuaba entre 18 y 80 aproximadamente. No se incluyeron en el estudio interacciones con niños o adolescentes. Género, edad y clase social son tres factores que ameritarían ser examinados en un estudio futuro más amplio.

Las grabaciones fueron transcritas empleando algunas de las convenciones de Gail Jefferson (en Atkinson y Heritage, 1984) y otras. Las transcripciones de interacciones londinenses fueron comprobadas por un hablante nativo de inglés londinense.

\section{PSIs de variación sociocultural en el presente estudio}

Se identificaron cinco PSIs o dimensiones principales con respecto a los cuales se puede distinguir el comportamiento de los londinenses del de los sevillanos del presente estudio:

Orientación a/hacia

- la formalidad o informalidad

- la expresión de consenso frente a la auto-afirmación

- una comunicación diádica y privada o gregaria y pública y, relacionado con esto,

- la reserva o locuacidad en la comunicación que reflejan diferentes grados de confian$z a ; y$, finalmente,

- la comunicación directa o indirecta en la transacción

Detallamos e ilustramos estas dimensiones a continuación. Antes hay que resaltar, sin embargo, que aquí nos referimos a tendencias o preferencias por ciertos comportamientos y no a comportamientos excluyentes.

\subsection{Orientación hacia la (in)formalidad}

Esta orientación se manifiesta en el contexto examinado en la preferencia de un grupo por el uso de formas convencionales y de formas innovativas por el otro. En el contexto del presente estudio, este parámetro correspondería a lo que Spencer-Oatey y Jiang (2003) denominan routinization-novelty o al de verbal routines-ad hoc formulation de House (1996; 2000) y se refiere a la orientación al uso de formas convencionales o rutinarias tales como fórmulas de cortesía frente al uso de formas menos fijas y más variadas. Se encontró un uso mayor de formas convencionales en los datos londinenses, particularmente en los comienzos y terminaciones de las interacciones. En las interacciones españolas, en cambio, se observa mayor flexibilidad y originalidad. 
Los comienzos y terminaciones son secciones que han sido identificadas como cruciales en cuanto facilitan el ingreso y la salida de una interacción (Laver, 1975; 1981). De hecho, se ha descrito la ocurrencia de intercambios de comunicación fática tales como saludos y despedidas como "rituales de acceso" (access rituals) (Goffman, 1971) que se emplean para la transición de la no interacción hacia la interacción y viceversa, mecanismos que a la vez ayudan a establecer, mantener y reforzar las buenas relaciones. En el área de la atención al público (service encounters) se ha descrito patrones de comienzos y terminaciones que se pueden distinguir claramente del cuerpo de la interacción y se ha resaltado el aspecto ritual de los mismos. Por ejemplo, Kuiper y Flindall (2000) proponen con referencia a interacciones entre clientes y cajeros en dos supermercados en Christchurch (Nueva Zelanda) que "[t]he beginning and end of conversations have a strong tendency to be formulaic because what needs to be done in them is a matter of social ritual" (Kuiper y Flindall, 2000: 188).

Con respecto a interacciones en farmacias, se esperaría posiblemente también encontrar comienzos y terminaciones rituales exceptuando posiblemente casos de emergencia donde por las prisas intercambios de saludos pueden ser obviados. Sin embargo, como vamos a ver, esto no necesariamente se da en las interacciones sevillanas mientras que sí ocurre con más frecuencia en las londinenses. En la siguiente tabla se puede apreciar precisamente que en las interacciones en Londres, se da un porcentaje más alto de comienzos y terminaciones rituales que en Sevilla, y que en Sevilla, particularmente en los comienzos, se emplean más mecanismos no rituales que rituales.

\begin{tabular}{|c|c|c|c|c|}
\hline & \multicolumn{2}{|c|}{ Comienzos } & \multicolumn{2}{c|}{ Terminaciones } \\
\hline & $\begin{array}{c}\text { Comienzos } \\
\text { rituales }\end{array}$ & Otros comienzos & $\begin{array}{c}\text { Terminaciones } \\
\text { rituales }\end{array}$ & Otras terminaciones \\
\hline Sevilla & $38 \%$ & $62 \%$ & $42.5 \%$ & $58.5 \%$ \\
\hline Londres & $77.5 \%$ & $22.5 \%$ & $97.5 \%$ & $3.5 \%$ \\
\hline
\end{tabular}

Tabla 1 . Uso de formas rituales y no rituales en Londres y Sevilla

En cuanto a comienzos, las formas rituales empleadas en Londres y que aparecen en un $77.5 \%$ de las interacciones, incluyen saludos (ej. hello, good morning, etc), y preguntas sobre el bienestar de la persona (ej. are you all right?, how are you?, etc.), que con frecuencia ocurren en forma de pares adyacentes, como en el siguiente ejemplo":
(1) (Londres) $(\mathrm{F}=$ farmacéutico; $\mathrm{C}=$ cliente)
$01 \mathrm{C}$ good morning
$02 \mathrm{~F}$ good morning how are you?
$03 \mathrm{C}$ uh yes fine yes 
En las respuestas a preguntas sobre el bienestar de la persona, como en el turno 4 de este ejemplo, se puede ver el carácter rutinario de estas preguntas. No parece esperarse nada más que un all right, fine o not too bad.

También se encontraron algunas ofertas de ayuda que pueden ocurrir con un agradecimiento rutinario producido por el/la cliente o seguidos de la petición del producto, como en el siguiente ejemplo:

(2) (Londres)

$01 \mathrm{~F}$ hello can I help?

$02 \mathrm{C}$ yeah do you have razors in here?

Con respecto a terminaciones, se encontró que, como indica la Tabla 1, el 97.5\% de las interacciones londinenses presentaba un patrón fijo: la ocurrencia de una fórmula de agradecimiento y/o un saludo de despedida. Es de interés destacar que se puede encontrar más de un ejemplo de las fórmulas de agradecimiento dentro de las terminaciones londinenses:

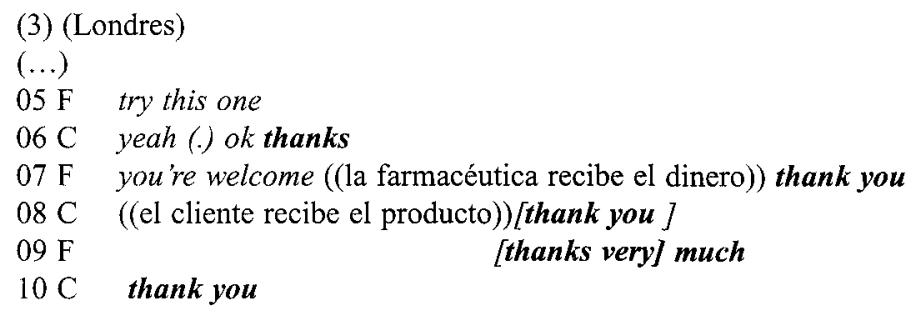

Estos resultados estarían de acuerdo con los de House (cfr. House, 1996; House, 2000), que ha encontrado que los hablantes anglófonos, en contraste con los hablantes de alemán, en sus estudios demuestran una preferencia por formas rutinarias.

En el caso de Sevilla, como se ha comentado, no se han encontrado patrones fijos en la misma medida, es decir, se ha encontrado una mayor variedad de formas. En cuanto a saludos, aparecen solamente en 16 ejemplos $(38 \%)$ y junto con una respuesta ritual como en el ejemplo (4), solamente en 8 interacciones de las 42 .

(4) (Sevilla)

$01 \mathrm{C}$ buenos días

$02 \mathrm{~F}$ hola::

En otras ocasiones, los clientes producen el saludo pero continúan con la petición, es decir, sin dar espacio para una respuesta:

(5) (Sevilla)

$01 \mathrm{C}$ hola (.) quiero un termalgín y un gelocatil no me digas que es lo mismo pero es que hay gusto hasta para las medicinas en mi casa

En las interacciones sevillanas, en la ausencia de saludos o preguntas por el bienestar de la persona, pueden darse otros elementos de comunicación fática o small talk (Coupland, 2000), es decir sin una transición gradual, como en el ejemplo (6) a continuación. Esto último se da en el $30.9 \%$ de las interacciones. 
(6) (Sevilla)

$01 \mathrm{C}$ me da dodotis

En cuanto a terminaciones en Sevilla, los patrones fijos tampoco se encuentran con la misma frecuencia que en las interacciones londinenses. Como indica la Tabla 1 más arriba, sólo el $45.2 \%$ de las interacciones incluye un saludo de despedida, y sólo el 19\% de estas interacciones incluye también una expresión de agradecimiento ${ }^{12}$. En una buena parte de las interacciones se cierra la interacción con el pago:

(7) (Sevilla)

$01 \mathrm{~F}$ te tomas dos dos y dos

$02 \mathrm{C}$ vale

$03 \mathrm{~F}$ tres noventa y cinco

((cliente paga y se va))

El uso mayor o menor de formas convencionales contribuye a dar a la interacción un carácter más o menos (in)formal, pudiéndose describir las interacciones londinenses como tendientes al polo formal y las sevillanas tendientes al polo informal. Esto se puede ver también en las selecciones léxicas, como el uso del farmacéutico de "tú" en el ejemplo (7) que crea acercamiento, al igual que en el uso más frecuente de marcadores del discurso como "sabes" en el ejemplo (8), con el que el cliente asume un punto de vista compartido (common ground) con el vendedor y contribuye a dar a la interacción transaccional el carácter de una conversación entre amigos.

(8) (Sevilla)

(...)

$37 \mathrm{C}$ éstas qué precio tienen porque yo me tengo que poner una [cada cada veinte] minutos porque yo me lavo mucho las manos

$38 \mathrm{~F}$ [dos ochenta] éstas son más baratas $=$

$39 \mathrm{C}=$ cuál (.) éstas? pues dame las más baratas porque // me las voy a poner aquí mismo (.) sabes?

Por otro lado, la orientación al uso menor de formas convencionales de los sevillanos sería parte de lo que algunos autores (cfr. Thurén, 1988; Fant, 1989; Bravo, 1999; Hernández Flores, 1999) destacan como un rasgo de los españoles de preferir mostrarse como originales y de destacar sus propias cualidades. Esto iría de acuerdo con la sugerencia inicial de Fant (1989: 251) de que las culturas hispánicas muestran una preferencia por "divergent manner for a dialectic atmosphere to emerge, or else the conversation will be perceived as dull and uninteresting". Esta preferencia estaría relacionada con la de "auto-afirmación" que consideramos en el siguiente apartado.

12 En Sevilla, se tiende a encontrar fórmulas de agradecimiento cuando el servicio prestado por el farmacéutico va más alla de los servicios rutinarios de una farmacia, como consejo brindado o tiempo adicional que el farmacéutico ha dedicado al cliente. Al respecto, véase también Hickey (1991). 


\subsection{Orientación hacia el consenso o la auto-afirmación}

Fant (1989) contrasta el comportamiento de escandinavos y españoles y sugiere, entre otras cosas, que mientras los primeros se orientan a la búsqueda de consenso, los segundos valoran la expresión de auto-afirmación. La noción de auto-afirmación proviene de Thurén (1988: 299) quien la define como una disposición de los españoles en su estudio (en Valencia) que se manifiesta mediante el "énfasis piscológico en la persona y su fuerza personal" (nuestra trad.). De acuerdo a esta autora, es importante para los españoles expresar las opiniones que tengan, aunque sean de desaprobación, con fuerza y de manera persuasiva. EI no hacerlo se consideraría una falta de sinceridad (véase también Bravo, 1999). Este tipo de comportamiento que incluye expresión de desacuerdo y contradicción se observa también en las interacciones en Sevilla mientras que en Londres la tendencia parece ser la de orientarse al acuerdo o al menos a no manifestar disenso. Así pues, mientras no se encontraron ejemplos de desacuerdo o contradicción en las interacciones londinenses, se identificaron 39 casos en los datos sevillanos los mismos que aparecen en respuesta a un consejo recibido o al punto de vista expresado por el interlocutor. En los turnos 7-13 de la interacción (9) a continuación, se puede observar, por ejemplo, intercambios donde se da un tipo de contradicción de manera reiterada ("te dije X" y "no me has dicho X").
(9) (Sevilla)
$01 \mathrm{~F}$ a quién
$02 \mathrm{C}$ a mí (.) Fastum (.) tienes?
$03 \mathrm{~F} \quad$ Fastum crema
(...)
$04 \mathrm{C}$ pero esto es para para:: la regla ((señalando la crema))
$05 \mathrm{~F}$ eso es para un dolor [es un antiinflamatorio]
$06 \mathrm{C} \quad$ [no pero en pastillas tienes?]
$07 \mathrm{~F}$ ah que te había dicho crema y te había [entendido]
$08 \mathrm{C}$
$09 \mathrm{~F} \quad=y a$ está que te he dicho
[ah no me] he enterado ((risas))
(...)
$10 \mathrm{~F}$ es que te dije::
$11 \mathrm{C}$ no me he enterado y ()
$12 \mathrm{~F}$ es que te dije::=
$13 \mathrm{C}=$ no me he enterado y (a ver si me llega)
(..)
$14 \mathrm{~F}$ siete cincuenta
$15 \mathrm{C}$ no bueno pues dejo entonces me llevo las pastillas ()

$\mathrm{El}$ análisis de las interacciones sevillanas de este estudio en contraste con las londinenses sugiere entonces que hay un grado más alto de tolerancia a la expresión de desacuerdo, tal como Fant (1989) había propuesto en la comparación que realizó entre escandinavos y españoles. Lo que dice sobre los españoles parece aplicarse también al presente estudio: "Hispanic speakers will simply tolerate a much higher degree of disagreement without incurring the risk of conversational breakdown" (Fant, 1989: 251). En trabajos más recientes, otros autores han llegado a conclusiones similares. Por ejemplo, Martínez Camino y Dalley (2004) analizaron discusiones en un foro electrónico en España (Cantabria) y los Estados 
Unidos (Michigan) y encontraron que los españoles expresan desacuerdo con mucha más frecuencia que el grupo anglosajón.

\subsection{Orientación hacia la comunicación diádica y privada o gregaria y pública}

Thurén (1988) destaca la disposición de los españoles en su estudio hacia la "sociabilidad" o disfrute de la compañía de otros en contraste con lo que ella denomina la preferencia por la conversación privada que se puede encontrar en el norte de Europa. Con referencia a tiendas, la sociabilidad de Thurén tendría el elemento de participación grupal que adicionalmente muchas veces vendría a ser un tipo de "actuación pública" (ver Fant, en Placencia, en prensa) en cuanto Thurén destaca que las conversaciones en las tiendas son públicas y que la gente se siente en libertad de participar sin necesidad de ser presentado (Thurén, 1988: 117). Esto es algo que Placencia (en prensa) identifica también en interacciones de tiendas de barrio en Madrid en contraste con interacciones en tiendas similares en Quito en donde parece haber una preferencia por interacciones diádicas "privadas" entre el farmacéutico y el cliente.

En cuanto a las interacciones en farmacias del presente estudio, se encuentra también que los londinenses se limitan a la interacción diádica con el farmacéutico como en el ejemplo (10):

(10) (Londres)

(...)

$04 \mathrm{~F}$ the doctor did give you the antibiotic and the cough syrup

$05 \mathrm{C}$ yeah

$06 \mathrm{~F}$ the antibiotic might clear it

$07 \mathrm{C}$ (I've had) three bottles the last time he gave me a bottle I got it from

here ( ) Procodeine () I've bought myself two other bottles since

then

$08 \mathrm{~F}$ oh did you?

$09 \mathrm{C}$ yeah two other bottles

$10 \mathrm{~F}$ thank you

(...)

Los clientes sevillanos en cambio se sienten en libertad de tomar parte en interacciones con otros clientes mientras esperan que les atiendan o entre el farmacéutico y otros clientes, generándose una interacción grupal, como en el siguiente ejemplo donde un segundo cliente toma parte en una interacción en curso:

(11) (Sevilla) $(\mathrm{C} 1=$ Cliente $1 ; \mathrm{C} 2=$ Cliente 2$)$

$01 \mathrm{Cl}$ pues:: (.) tengo dolor de cabeza malestar general ((tos)) es que llevo tomando un montón de cosas y ya no sé ni lo que me voy a mmm

$02 \mathrm{~F}$ hombre si puede tomar por ejemplo Frenadol pero entonces no tienes por qué tomar esto

$03 \mathrm{C} 1$ no si esto yo lo tomo yo nada más que por la noche eso eso es nada más que para la tos una cucharada por la noche para dormir

$04 \mathrm{C} 2$ no le vaya a hacer a usted daño

$05 \mathrm{Cl}$ no (...) no:: para que que:: no de (le) fastidie la tos por la noche por lo menos que duerma 


\section{$06 \mathrm{C} 2$ y si no aspirina (o) el paracetamol}

$07 \mathrm{Cl}$ paracetamol es que estoy harta de tomar paracetamol yo ya creo que el paracetamol el hígado te lo machaca como un demonio y llevo ya tomando paracetamol por un tubo

$08 \mathrm{C} 2$ y el Visolgrín es muy bueno

$09 \mathrm{C} 1$ si pero esto también llevaría paracetamol seguramente

$10 \mathrm{C} 2$ y si no aspirina

(...)

En este ejemplo, como en otros, se puede ver que los clientes sevillanos se sienten a gusto dando sugerencias o consejos no solicitados ${ }^{13}$ (en negrita) a otros clientes y que dichos consejos son bien recibidos, es decir, no se consideran intromisiones en la privacidad del interlocutor.

Más aún, se puede apreciar también en el ejemplo (11) que, al igual que los clientes se sienten en libertad de dar un consejo, los que lo reciben también se sienten en libertad de rechazarlo sin ofender con esto al emisor del consejo (véase turnos 07 y 09). Estos resultados irian de acuerdo con las observaciones que hace Hernández Flores sobre la autoafirmación de españoles y la emisión de consejos y su recepción con referencia a otro tipo de contexto:

Since advice involves the utterance of the speaker's personal ideas, self-affirmation would stand for the engagement of the speaker with these ideas. Giving advice works as the speaker's (the advice giver) desire for self-affirmation because s/he is expressing a personal (and good, under her/his view) idea, whereas the hearer (the advice receiver) still has the right of accepting or rejecting advice, i.e., stressing her/his own competence in making a decision (self-affirmation). (Hernández Flores, 1999: 42)

Por otra parte, las interacciones pueden desarrollarse entre varios clientes, como en el siguiente ejemplo:

(12) (Sevilla)

$01 \mathrm{Cl}$ oye oye (qué bien) poder meterse aquí verdad.

$02 \mathrm{C} 2$ más calentito verdad

$03 \mathrm{C} 3$ tenéis que poner un cartel ahí cierren la puerta por favor

$04 \mathrm{Cl}$ e::xacto, exacto, exacto (.) si tuviéramos cuidado con cerrar la puerta [estaríamos mucho] mejor

$05 \mathrm{C} 2$ [estaríamos mucho] $=$

$06 \mathrm{Cl}=$ mejor

$07 \mathrm{C} 3$ pero es que el cambio que hay de aquí a fuera::

$08 \mathrm{Cl}$ yo siempre acostumbro a cerrar la puerta

$09 \mathrm{C} 3$ claro claro claro

$10 \mathrm{C} 1$ p'al estómago ((al farmacéutico, dándole la receta al mismo tiempo)) pero si no me duele pa' que lo voy a tomar

$11 \mathrm{~F}$ ya está (.) veinte céntimos

$12 \mathrm{Cl}$ a ver si ponéis el papelito para que cierren la puerta 
Intercambios como los de esta interacción constituyen ejemplos de comunicación fática que sugieren que los clientes en las interacciones sevillanas buscan establecer rapport (positivo) no sólo con el farmacéutico sino con otros clientes. Es interesante notar que en las interacciones Sevillanas intercambios de comunicación fática se dan en un $59.5 \%$ de las interacciones mientras que sólo ocurren en un $15 \%$ de las londinenses. En Londres, los ejemplos identificados en todo caso corresponden a interacciones diádicas entre un farmacéutico y un cliente y normalmente comienzan bajo la iniciativa del farmacéutico. Adicionalmente, en las interacciones sevillanas se ha encontrado un mayor número y una gama más amplia de activadades encaminadas a establecer rapport (positivo) incluyendo bromas como en el ejemplo (13) (turnos 5-7) más abajo, intercambios sobre salud, hábitos, el tiempo, problemas personales y experiencia, historia biográfica, e interacciones anteriores.
(13) (Sevilla)
$01 \mathrm{~F}$ a quién atiendo
$02 \mathrm{C} 1$ a mí ( ) de algodón [pero] de esos pequeñitos que sean buenos=
$03 \mathrm{~F} \quad$ [sí ]
$=$ [son todos] mu' buenos [no?]
$04 \mathrm{Cl}$ [son todos] [son todos iguales no?
$05 \mathrm{C} 2$ aquí todo es de calidad aquí todo es de calidad
((risas))
$06 \mathrm{C} 2$ menos los dueños todo es calidad
$07 \mathrm{C} 1$ vale gracias

En Londres en cambio, el rapport (positivo) parece establecerse más comúnmente mediante formas convencionales en los comienzos y terminaciones y los clientes no parecen sentirse en libertad de tomar parte en otras interacciones que no sean las suyas puesto que posiblemente serían calificados de entrometidos. Se pudo observar por ejemplo que en una ocasión la farmacéutica y un cliente estaban hablando informalmente del cabello del último y de secretos para conservarlo bien cuando se dieron cuenta de la presencia de otro cliente. El cliente que hablaba de su cabello pidió disculpas al otro cliente por haberle "causado molestias" con un tema tan poco importante. Desde la perspectiva de un español no habría habido necesidad de ofrecer disculpas.

Esta distinción entre la comunicación diádica, privada frente a la comunicación gregaria, pública estaría relacionada posiblemente con las nociones de individualismo-colectivismo (véase Hofstede, 1984) y las de independencia-interdependencia (Markus y Kitayama, 1991). En el área de la cortesía, como es sabido, una de las criticas que se ha hecho al modelo de Brown y Levinson (1987) es que la noción de imagen (negativa) que proponen refleja un individualismo que caracterizaría a las culturas anglosajonas pero que no necesariamente se aplica a otras donde la imagen del grupo y no la del individuo solamente es también importante (véase por ej. Matsumoto, 1988; Ide, 1989).

\subsection{Reserva o locuacidad en la comunicación}

Estrechamente relacionado con la sociabilidad de los españoles sugerida por Thurén (1988) estaría su orientación a ser comunicativos o locuaces. Esto entraría en contraste con 
la orientación hacia la reserva que parece caracterizar a los londineneses en las interacciones del presente estudio ${ }^{14}$.

De acuerdo a Thurén la disposición sociable del individuo se manifiesta esencialmente mediante la comunicación verbal y el no ser comunicativo para los españoles en su estudio sería interpretado como ser "poco amigable" (unfriendly) (Thurén, 1988: 222). A la persona que pone distancia se la calificaría de tímida e insociable.

La locuacidad de los sevillanos se observaría en la mayor ocurrencia de intercambios de comunicación fática grupales como los discutidos en 4.3 al igual que en comentarios que los clientes hacen al farmacéutico que no son esenciales para la transacción y con los que comunican algún tipo de información personal. Así en la interacción (5) más arriba, la cliente comenta que "... pero es que hay gusto hasta para las medicinas en mi casa" luego de indicar el producto que quiere, y en la (12) más arriba también, la cliente pide con una receta algo para el estómago (turno 10) y añade su opinión en el mismo turno "pero si no me duele pa' que lo voy a tomar" casi como si estuviera pensando en voz alta. En la siguiente interacción nuevamente se observa este tipo de comentarios (en negrita):

(14) (Sevilla)

(..)

$40 \mathrm{~F}$ aparte tú bebes agua o no

$41 \mathrm{C} 2$ es que eso también se lo he comentado [a ella

$43 \mathrm{Cl}$

[bebe muy poco agua $=$

$44 \mathrm{C} 2$ =muy poca

$45 \mathrm{~F}$ tú eres de beber [agua

$46 \mathrm{C} 2$ [eso se lo he dicho yo a mi madre es que bebo muy poquísima agua

(...)

Comentarios de éstos serían ejemplos del gusto por el "despilfarro" o "exceso verbal" del que hablan Narbona y otros (1998: 210-1) en su obra sobre el español oral de Andalucía y que según ellos "... cumple un claro papel socializador, reforzador de la solidaridad" (1998: 211). Los londinenses en este estudio en cambio parecerían orientarse más hacia el "ahorro" verbal noción que Narbona y otros (1998: 210) contraponen a la de de despilfarro o derroche ${ }^{15}$. Se puede ver esto en el cuerpo de la siguiente interacción:

(15) (Londres)
$01 \mathrm{C} \quad$ hello
$02 \mathrm{~F} \quad$ hello
$03 \mathrm{C} \quad$ can I have some () please
$04 \mathrm{~F} \quad$ which kind $=$
$05 \mathrm{C} \quad=$ the small one please $=$

14 Distinciones relacionadas aparecen, por ejemplo, en Bailey (1997), quien describe el estilo reservado (restrained) de los coreanos frente al estilo efusivo (de involvement) de los afro-americanos en tiendas de licores en su estudio, y en Márquez Reiter y Placencia (2004) con respecto a interacciones en tiendas de ropa y accesorios en Montevideo y Quito donde se observa que los vendedores montevideanos son más comunicativos que los quiteños.

15 En términos de Grice (1975), este contraste podría expresarse como una tendencia a observar la máxima de cantidad por parte de los ingleses, y a no observarla, por parte de los sevillanos. 
$06 \mathrm{~F}=$ thank you

((farmacéutico busca producto))

$07 \mathrm{~F}$ that's two forty-nine please

((cliente paga))

$08 \mathrm{~F}$ your change thank you

$09 \mathrm{C}$ thank you

En la siguiente interacción en Sevilla por otro lado se encuentra un ejemplo claro de una cliente que proporciona información personal no solicitada y no esencial para la transacción. Esto se puede observar en los turnos 11,19 y 25 :

(16) (Sevilla)

(...)

$05 \mathrm{C}$ :: mira (.) que quería una botellita de agua de rosas=

$06 \mathrm{~F}$ de agua de rosas

$07 \mathrm{C}$ sí mire que tengo una quemadura en la mano que no sé que ponerme

$08 \mathrm{~F}$ a ver

$09 \mathrm{C}$ te la enseño?

$10 \mathrm{~F} \quad($ )

$11 \mathrm{C}$ es que fue un [golpe de Nochebuena] yo qué sé con lo que fue pero que $\mathrm{me}=$

$12 \mathrm{~F}$ [con qué fue?]

$13 \mathrm{C}=$ duele mira ves

$14 \mathrm{~F}$ claro claro claro

$15 \mathrm{C}$ una pomada?

(...)

$19 \mathrm{C}$ y una TIRITA PEQUEÑA si me lo protejo que tenga el ancho de esto (.) esto fue anoche no/ fue la noche de Nochebuena/ que tuve una nieta en casa que se me puso mala con fiebre

$20 \mathrm{~F}$ una tirita así no?

$21 \mathrm{C}$ no pero que sean chicas pero anchitas yo tengo en casa tiritas de ancho de esto

$22 \mathrm{~F}$ no pero digo para ponértela ahora

$23 \mathrm{C}$ si:: para ahora que es que si no

$24 \mathrm{~F}$ este es el suyo (.) esto es para cortarlo se despegan muy fácilmente y se ponen

$25 \mathrm{C}$ por qué no me cortas una y ya no me llevo eso y lo tengo yo allí guardado para estos cortes (.) es que mi nieta estaba en mi casa, $\mathbf{y}$ a las seis empezó con treinta y siete a las ocho tenía treinta y ocho y pico y a las ocho y media nueve menos cuarto treinta y nueve de fiebre así que dije a ver que haga algo a la pobre mi hija se llevó a las otras dos () y me hice la herida () ahí te he puesto para que te cobres el agua de rosas

El proporcionar información personal puede describirse como una estrategia de acercamiento en cuanto constituye un tipo de auto-revelación (self-disclosure) ${ }^{16}$ y parece ser a la vez una forma de reflejar y recrear relaciones de confianza. Es decir, al dar información

16 Nos atenemos aquí a la definición general de Hargie et alii (1994: 218) de self-disclosure como la revelación del hablante a otros de información personal. 
personal no directamente pertinente para la transacción los sevillanos parecen tratar al farmacéutico ( $u$ otros clientes) como alguien con el que tienen una relación personal de confianza (por ej., amigo/a) y no sólo de carácter institucional (farmacéutico-cliente) mientras que los clientes ingleses parecen ajustarse mayormente al rol institucional. Al hablar de confianza, nos referimos aquí a la noción que Thurén (1988) y Fitch (1994) resaltan y que ha sido explorada en diferentes contextos españoles (cfr. Fant, 1989; Bravo, 1999; Hernández Flores, 2004) e hispanoamericanos (cfr. Moles, 1974; Fitch, 1994). Dicha noción transmite la idea de cercanía (closeness) y familiaridad (cfr. Thurén, 1988: 222) al igual que la de mayor libertad de comportamiento como lo sugiere Fitch (1994: 197) para quien,

[t]he greater the confianza that exists, the greater the behavioural latitude in interaction, for example in joking, amount and kind of information about the self made accessible, and giving directives.

Parte de la expresión de confianza constituiría entonces el proporcionar información personal al igual que el sentirse en el derecho de dar consejos a otros clientes y de expresar desacuerdo libremente.

La locuacidad de los sevillanos parece estar conectada con estrategias de acercamiento, mientras que el ahorro verbal de los londineneses con estrategias de distanciamiento o lo que Márquez Reiter y Placencia (2004) denominan respectful distance. Esto estaría de acuerdo con lo que algunos autores han propuesto sobre la orientación relativa en la cortesía de los ingleses hacia el polo negativo dentro del marco de Brown y Levinson (cfr. Sifianou, 1989 y 1992; Hickey, 1991; Vázquez Orta, 1995; Valeiras Viso, 2002), y la de los españoles hacia el polo positivo (cfr. Hickey, 1991; Vázquez Orta, 1995; Lorenzo-Dus, 2001; Valeiras Viso, 2002).

\subsection{Orientación a la comunicación (in)directa en la transacción}

Bajo este principio, nos referimos a la forma en la que se produce la formulación del ruego que Turnbull (2003) asocia con orientación hacia la eficiencia "transaccional" o "interpersonal". Para Turnbull, la eficiencia transaccional requiere de brevedad de acuerdo al principio del menor esfuerzo, al igual que claridad, mientras que la segunda por implicación estaría caracterizada por mayor esfuerzo y menos claridad. Turnbull ilustra esta distinción con un ejemplo de ruegos y sugiere que si la persona que hace el ruego adopta una orientación transaccional el resultado será una formulación franca (blunt) y directa como "put this box in my car" (Turnbull, 2003:107). La eficiencia interpersonal por otro lado requeriría la producción de una forma elaborada con atributos como la vaguedad y la inclusión de disculpas como en "I'm sorry to have to bother you but I have to get this box into my car but it is too heavy for me to lift alone and I'd be really grateful if it wouldn't be too much trouble" (2003: 107). Turnbull sin embargo, resalta que para conseguir una meta transaccional es necesario atender a aspectos interpersonales también (véase también Leech, 1983; Aston, 1988). En realidad, como dice Turnbull (2003: 108), “... transactional goals will get accomplished only if interpersonal goals are attended to". Sin embargo, puede haber variación situacional y cultural en cuanto al grado de atención que se da al aspecto interpersonal en la consecución de una meta transaccional (cfr. Placencia, en prensa), el 
punto en la interacción donde se manifiestan la meta interpersonal al igual que las formas que se emplean en su realización (formas rituales o individualizadas).

Siguiendo el marco de análisis de ruegos de Blum-Kulka et alii (1989), clasificamos los primeros ruegos de cada interacción en directos, convencionalmente indirectos y no-convencionalmente indirectos, como en los siguientes ejemplos:

(17) (Sevilla)

C eh: dame linemín y somacina (ruego directo)

(18) (Londres)

C hi uh can I have patches for smokers? the small ones (ruego convencionalmente indirecto)

(19) (Londres)

C hello I left a prescription here yesterday (ruego no convencionalmente indirecto)

Añadimos una cuarta categoría de ruegos ("no verbales") en los que el cliente hace el ruego entregando una receta al farmacéutico:

(20) (Londres)
$01 \mathrm{C}$ good morning
$02 \mathrm{~F}$ good morning how are you
$03 \mathrm{C}$ uh yes fine yes
$04 \mathrm{~F}$ yes
$((\mathrm{el}$ cliente le da la receta al farmacéutico))
$05 \mathrm{C} \quad$ yes

La siguiente tabla resume los resultados sobre tipo de ruego identificado en los dos contextos socioculturales:

\begin{tabular}{|c|c|c|c|c|}
\hline Lugar & Directos & $\begin{array}{c}\text { Convencionalmente } \\
\text { indirectos }\end{array}$ & $\begin{array}{c}\text { No convencionalmente } \\
\text { indirectos }\end{array}$ & No verbales \\
\hline Sevilla & $54.8 \%$ & $14.3 \%$ & --- & $30.9 \%$ \\
\hline Londres & $2.5 \%$ & $50 \%$ & $7.5 \%$ & $32.5 \%$ \\
\hline
\end{tabular}

Tabla 2. Formas de ruegos ${ }^{17}$

Dejando de lado los ruegos no verbales, se puede ver que los sevillanos muestran una preferencia por formas directas, y los londinenses por formas indirectas. No se da ningún caso de indirección no convencional en los datos sevillanos y si unos pocos en los londineneses. Estos resultados están de acuerdo con los de otros estudios sobre el español peninsular como los de Lorenzo y Bou (2003) y Placencia (en prensa) en los que se encuentra un predominio en el uso de formas directas, pero no así con los de otros autores como Vázquez 
Orta (1995), Le Pair (1996) y Díaz Pérez (1999), entre otros, quienes encuentran que en las situaciones que examinaron predomina globalmente el uso de formas convencionalmente indirectas. Hay que tener en cuenta que las situaciones que estos últimos autores estudiaron no incluyen interacciones de atención al público y que se basan en datos de juegos de roles o cuestionarios que no necesariamente reflejan usos reales. En cuanto al inglés, los resultados de este estudio estarían de acuerdo con otros sobre el inglés británico como el de Vázquez Orta (1995), Díaz Pérez (1999) y Márquez Reiter (2000).

\section{Motivaciones detrás de las preferencias de interacción}

En esta sección intentamos explicar con respecto a varios factores las diferencias en la orientación de sevillanos y londinenses con respecto a los principios o dimensiones considerados en la sección anterior. Estamos de acuerdo con la propuesta de Spencer-Oatey (en prensa) y Spencer-Oatey y Jiang (2003), de que el modo de conducir las relaciones interpersonales no estaría motivado por consideraciones de imagen solamente, sino también por consideraciones de los derechos y obligaciones que van con la actividad, al igual que las metas que los participantes persiguen en la interacción, aunque no siempre es fácil distinguir unas motivaciones de otras.

En cuanto a consideraciones de imagen, en rasgos generales se puede observar que mientras los sevillanos prestan atención al grupo (otros clientes), los londinenses se concentran en la díada o sea que la imagen grupal puede ser de importancia en un caso pero no en el otro. Por otro lado, mientras los primeros parecen enfatizar la expresión de camaradería en la interacción mediante el empleo de formas espontáneas, trato informal, ruegos directos y la comunicación de información personal, los segundos parecen enfatizar la expresión de consideración y respeto por medio de intercambios rituales y el uso de convencionalismo indirecto. Se trata en ambos casos de estrategias encaminadas a que la interacción sea satisfactoria pero utilizando mecanismos diferentes. Y por último, se puede decir que parece haber más preocupación por la auto-imagen (véase Chen, 2001) que la del interlocutor en las interacciones sevillanas lo que se manifiesta en la expresión de auto-afirmación y el mayor uso de formas espontáneas con lo que los sevillanos mostrarían su originalidad. Los londinenses por su lado parecen enfatizar la imagen del otro al evitar inmiscuirse en su territorio y no dar consejos a otros clientes, por ejemplo, y al mantener distancia mediante el uso de formas convencionales y el hecho de que se restringen a temas pertinentes para la transacción solamente.

Con respecto a la noción de derechos y obligaciones, ésta se refiere a las expectativas de los participantes de lo que debe darse en la interacción en cuanto a los roles que cada participante desempeña. Aquí resulta útil la distinción de Spencer-Oatey (en prensa) entre comportamiento prescrito (prescribed) y permisible (permissible), entre otras distinciones. El comportamiento prescrito es similar en los dos contextos socioculturales en cuanto los clientes tienen derecho a solicitar un producto que se pueda encontrar en una farmacia, y los farmacéuticos la obligación de proporcionárselo a cambio de un pago (o la firma de un formulario si tienen derecho a medicina gratuita). Un aspecto de variación está, sin embargo, en lo que es permisible. Los sevillanos, por ejemplo, pueden tomar parte en las interacciones de otros clientes, dándoles su opinión o consejos y el/la receptor/a de los mismos puede rechazarlos sin que nada de esto constituya una intromisión o un acto amenanzante. Esto 
no parece ser permisible en las interacciones londinenses. Por otro lado, el uso de fórmulas rutinarias de agradecimiento que se comentó era poco frecuente en las interacciones sevillanas y más frecuente en las londinenses sería una indicación de cómo los participantes perciben la transacción, como algo a lo que los unos (los sevillanos) tienen pleno derecho (un intercambio igualitario) mientras que para los londinenses parece representar un cierto grado de imposición.

Finalmente, en cuanto a las metas de los participantes, se da por sentado que las metas que pueden perseguir son múltiples y que no se restringen necesariamente a la transacción (véase por ej. Coupland, Coupland y Robinson, 1992). En las interacciones examinadas en este estudio se observa que tanto sevillanos como londinenses persiguen además de una meta transaccional, metas relacionales/interaccionales, buscando construir buenas relaciones con sus interlocutores. Como se observó anteriormente, los mecanismos que emplean son los que varían. Igualmente, el punto en la interacción en el que se orientan a actividades relacionales parece estar sujeto a variación. Así, se observó que los sevillanos pueden orientarse de entrada a la tarea produciendo su ruego en el primer turno de la interacción y pasando luego a actividades relacionales no convencionales. En contraste, se observó que en las interacciones londinenses se atiende a aspectos relacionales principalmente en los comienzos y finales de las interacciones empleándose mecanismos rituales. Adicionalmente, una variación de tipo más global que se puede observar es que a diferencia de los londineneses, algunos de los sevillanos parecen tratar la interacción en la farmacia como una ocasión para socializar con otros clientes, corroborando la observación de Thurén (1988:117) sobre tiendas en Valencia de que "[t]he store conversations are defined as friendly occasions, which means that you behave as if you take it for granted that everyone is friendly ..." y que " $t]$ he store conversation is an arena for weaving friendships and alliances ..." Sin embargo, consideramos que sería necesario estudiar este fenómeno en más detalle con respecto a la variable de edad, por ejemplo, pues es posible que se dé más frecuentemente en Sevilla entre personas de mayor edad y que también se dé en Londres entre dichas personas.

\section{Resumen y observaciones finales}

En este estudio examinamos aspectos de variación en los modos de conducir las relaciones interpersonales en interacciones en farmacias en Sevilla y Londres, apoyándonos principalmente en las propuestas de Spencer-Oatey (en prensa) y Spencer-Oatey y Jiang (2003) sobre la GI y los PSIs, al igual que en los estudios transculturales de Fant (1989) y House (cfr. House 1996; House 2000), entre otros que destacan dimensiones o principios de variación sociocultural.

La orientación con respecto a los PSIs que los sevillanos y londinenses manifiestan tener en las interacciones examinadas en el presente estudio se resumen así: 


\begin{tabular}{|lll|}
\hline Sevillanos & Londinenses \\
\hline informalidad & $\leftarrow \rightarrow$ & formalidad \\
auto-afirmación & $\leftarrow \rightarrow$ & consenso \\
comunicación gregaria y pública & $\leftarrow \rightarrow$ & comunicación diádica y privada \\
locuacidad en la comunicación & $\leftarrow \rightarrow$ & reserva en la comunicación \\
comunicación directa & $\leftarrow \rightarrow$ & comunicación indirecta \\
\hline
\end{tabular}

Tabla 3. PSIs para los sevillanos y los londinenses

Siguiendo a Spencer-Oatey y Jiang (2003) y Spencer-Oatey (en prensa) se interpretaron estas diferencias en orientación con respecto a variación entre sevillanos y londinenses en cuanto a preocupaciones de imagen, derechos y obligaciones asociados con la actividad y las metas que persiguen los participantes en la interacción. Así, por ejemplo, se encontró preocupación por la imagen grupal en las interacciones sevillanas y no en las londinenses. Igualmente se encontró que mientras los sevillanos se orientan al uso de estrategias de acercamiento, los londinenses parecen optar por estrategias que les permiten mantener distancia, mostrando respeto por la privacidad y autonomía de su interlocutor (o posibles interlocutores).

En cuanto a derechos y obligaciones, se observó que hay variación en cuanto a lo que es comportamiento permisible en un caso y otro y en cuanto a cómo los participantes perciben la transacción, como un intercambio igualitario los sevillanos y como una acción que representa cierto grado de imposición para los londinenses. $Y$ finalmente, sobre metas que persiguen los participantes, se encontró que tanto sevillanos como londinenses demuestran atender a metas transaccionales y relacionales; sin embargo parecen hacerlo en diferente grado, diferentes puntos de la interacción y utilizando mecanismos distintos.

$\mathrm{Si}$ bien hemos encontrado útiles las propuestas de Spencer Oatey (en prensa) y Spencer-Oatey y Jiang (2003) en cuanto a las motivaciones que hay detrás del uso de diferentes estrategias en la GRI, consideramos que ciertos fenómenos lingüísticos identificados en este estudio se pueden explicar también teniendo en cuenta consideraciones que van más allá del nivel micro de análisis. Así, por ejemplo, la auto-afirmación de los españoles que numerosos autores han identificado y que emerge también en el presente estudio, podría estar relacionada con desarrollos socio-políticos en España: el cambio de régimen de la dictadura a la democracia en los años 70 llevó a los españoles que por décadas habían estado sometidos a censura al período conocido como "el destape" en el que se volcaron a la expresión libre sobre política, sexo, derechos sociales y otras áreas, y que su aprecio por la expresión libre de opiniones se extendió también a la comunicación en la vida diaria. Consideraciones de este tipo están fuera del alcance del presente estudio pero las mencionamos en cuanto creemos que es importante no perder la perspectiva del contexto más amplio en el que están enmarcadas las interacciones de la vida cotidiana.

Para concluir, quisiéramos resaltar que, tal como habíamos indicado en la introducción, el presente es un estudio exploratorio, basado en un número limitado de interacciones y que por tanto, un trabajo más amplio, en el que se tomen en cuenta diferentes variables (ej. edad, género, clase social) sería un siguiente paso. También consideramos que sería impor- 
tante complementar el estudio con entrevistas a clientes y farmacéuticos para profundizar en las motivaciones detrás del uso de las diferentes estrategias en la GI identificadas en el presente estudio.

\section{Referencias bibliográficas}

Aronson, K. y B. Rundström (1989): "Cats, dogs, and sweets in the clinical negotiation of reality: on politeness and coherence in pedriatric discourse", Language in Society, 18, págs. 483-504.

Aston, G. (1988): Learning Comity. An Approach to the Description and Pedagogy of Interactional Speech. Bologna, CLUEB.

Atkinson, J. M. y J. Heritage (1984): “Transcript notation". En Atkinson, J. M. y J. Heritage (eds.): Structures of Social Action. Studies in Conversation Analysis. Cambridge, Cambridge University Press, págs. ix-xvi.

Bailey, B. (1997): "Communicating respect in interethnic service encounters", Language in Society, 26, págs. 327-356.

Blum-Kulka, S. S. (1987): "Indirectness and Politeness in Requests: Same or Different?", Journal of Pragmatics, 11, págs. 131-46.

Blum-Kulka, S., J. House y G. Kasper (eds.) (1989): Cross-Cultural Pragmatics: Requests and Apologies. Norwood, N.J., Ablex.

Bravo, D. (1999): “¿Imagen 'positiva' vs 'imagen negativa'? Pragmática sociocultural y componentes de face", Oralia. Análisis del Discurso Oral, 2, págs. 155-184.

Brown, P. y S. Levinson (1987): Politeness. Some Universals in Language Use. Cambridge, Cambridge University Press.

Brown, G. y G. Yule (1983): Discourse Analysis. Cambridge, Cambridge University Press.

Carrasco Santana, A. (1999): "Revisión y evaluación del modelo de cortesía de Brown y Levinson", Pragmalinguística, 7, págs. 1-44.

Coupland, J. (2000): Small Talk. London, Longman.

Coupland, J., N. Coupland y J. D. Robinson (1992): "“How are you?': Negotiating phatic communion”, Language in Society, 21, págs. 207-230.

Culpeper, J. (1996): “Towards an anatomy of impoliteness", Journal of Pragmatics, 25, págs. 349-367.

Culpeper, J., D. Bousfield y A. Wichmann (2003): "Impoliteness revisited: with special reference to dynamic and prosodic aspects", Journal of Pragmatics, 35, págs. 1545-579.

Chen, R. (1993): "Responding to compliments. A contrastive study of politeness strategies between American English and Chinese speakers", Journal of Pragmatics, 20, págs. 49-75.

Chen, R. (2001): "Self-politeness: A proposal", Journal of Pragmatics, 33, págs. 87-106.

Díaz Pérez, F. J. (1999): "Requesting in British English and Peninsular Spanish: A comparative analysis of politeness strategies". En Álvarez Benito, G. et alli (eds.): Lenguas en Contacto. Sevilla, Mergablum, págs. 171-180.

Eelen, G. (2001): A Critique of Politeness Theories. Manchester, St Jerome.

Fant, L. (1989): "Cultural mismatch in conversation: Spanish and Scandinavian communicative behaviour in negotiation settings", Hermes Journal of Linguistics, 3, págs. 247-265.

Fant, L. y L. Granato (2002): "Cortesía y gestión interrelacional: hacia un nuevo marco conceptual". Stockholm Studies in Interaction, Identity and Linguistic Structure II, 1-6, http://ab1.isp.su.se/iis/ Siiseng.htm, Universidad de Estocolmo.

Fitch, K. L. (1994): "A cross-cultural study of directive sequences and some implications for compliance-gaining research", Communication Monographs, 61, págs. 185-209.

Fraser, B. (1990): "Perspectives on politeness", Journal of Pragmatics, 14, págs. 219-236.

Fraser, B. y W. Nolen (1981): "The association of deference with linguistic form", International Journal of the Sociology of Language, 27, págs. 93-109. 
Garcés Conejos, P. (1993): "Revisión crítica de algunos de los postulados de la teoría de la cortesía linguística propugnada por Brown y Levinson", Quaderns de Filología, Estudis Linguistics, 1, págs. 43-62.

Goffman, E. (1971): Relations in Public: Microstudies of the Public Order. New York, Basic Books. Grice, H. P. ([1967]1975): "Logic and conversation". En Cole, P. y Morgan, J. L. (eds.): Syntax and Semantics, vol. 3: Speech Acts. New York, Academic Press, págs. 41-58.

Hargie, O., C. Saunders y D. Dickson (1994): Social Skills in Interpersonal Communication. London, Routledge.

Hernández Flores, N. (1999): "Politeness ideology in Spanish colloquial conversations: The case of advice", Pragmatics, 9 , págs. 37-49.

Hernández Flores, N. (2004): "Politeness as 'face' enhancement: An analysis of Spanish conversations between friends and family". En Márquez Reiter, R. y Placencia, M. E. (eds.): Current Trends in the Pragmatics of Spanish. Amsterdam, John Benjamins, págs. 265-284.

Hickey, L. (1991): "Comparatively polite people in Spain and Britain", ACIS, 4, págs. 2-6.

Hofstede, G. (1984): Culture's Consequences, International Differences in Work-Related Values. California, Sage.

House, J. (1996): "Contrastive discourse analysis and misunderstanding: The case of German and English". En Hellinger, M. y Ammon, U. (eds.): Contrastive Sociolinguistics. Berlin, Mouton, págs. 345-361.

House, J. (2000): "Understanding misunderstanding: A pragmatic-discourse approach to analysing mismanaged rapport in talk across cultures". En Spencer-Oatey, H. (ed.): Culturally Speaking. Managing Rapport through Talk across Cultures. London, Continuum, págs. 145-164.

Ide, S. (1989): "Formal forms and discernment: Two neglected aspects of universals of linguistic politeness", Multilingua, 8, págs. 223-248.

Janney, R. W. y H. Arndt (1993): "Universality and relativity in cross-cultural politeness research: A historical perspective", Multilingua, 12, págs. 13-50.

Kerbrat-Orecchioni, C. (1997): "A multilevel approach in the study of talk-in-interaction", Pragmatics, 7, págs. 1-20.

Kuiper, K. y M. Flindall (2000): "Social rituals, formulaic speech and small talk at the supermarket checkout”. En Coupland, J. (ed.): Small Talk. London, Longman, págs. 1-25.

Lakoff, R. (1976): "Why you can't say what you mean". Review of Edwin Newman, Strictly speaking", Centrum, 4, págs. 151-170.

Laver, J. (1975): "Communicative functions of phatic communion". En Kendon, A. Harris, R.M. y Key, M.R. (eds.): Organization and Behavior in Face-to-Face Interaction. The Hague, Mouton, págs. 215-238.

Laver, J. (1981): "Linguistic routines and politeness in greeting and parting". En Coulmas, F. (ed.): Conversational Routine: Explorations in Standard Communication Situations and Prepatterned Speech. The Hague, Mouton, págs. 289-304.

Leech, G. N. (1983): Principles of Pragmatics. London, Longman.

Le Pair, R. (1996): "Spanish request strategies: A cross-cultural analysis from an intercultural perspective", Language Sciences, 18, págs. 651-670.

Lorenzo-Dus, N. (2001): "Compliment responses among British and Spanish university students. A contrastive study", Journal of Pragmatics, 33, págs. 107-127.

Lorenzo, N. y P. Bou (2003): "Gender and politeness: Spanish and British undergraduates' perceptions of appropriate requests". En Santaemilia, J. (ed.): Género, lenguaje y traducción. Valencia, Universidad de Valencia/Dirección General de la Mujer, págs. 187-199.

Malone, M. J. (1997): Worlds of Talk. The Presentation of Self in Everyday Conversation. Cambridge, Polity Press.

Mao, L. R. (1994): “Beyond politeness theory: 'Face' revisited and renewed", Journal of Pragmatics, 21, págs. 451-86. 
Markus, H. R. y S. Kitayama (1991): "Culture and the self: Implications for cognition, emotion and motivation", Psychological Review, 98, págs. 224-253.

Márquez Reiter, R. (2000): Linguistic Politeness in Britain and Uruguay. Amsterdam, Benjamins.

Márquez Reiter, R. y M. E. Placencia (2004): "Displaying closeness and respectful distance in Montevidean and Quiteño Spanish". En Márquez Reiter, R. y Placencia, M. E. (eds.): Current Trends in the Pragmatics of Spanish. Amsterdam, John Benjamins, págs. 121-155.

Martínez Camino, G. y E. Dalley (2004): "Cortesía e ideología en el discurso: La atenuación de la discrepancia en los foros Webct". En Martínez Camino, G., García Casado y Scurfield, S. (eds.): Encuentro de lenguas y literaturas. Santander, Ediciones TGD, págs. 61-70.

Matsumoto, Y. (1988): "Reexamination of the universality of face: Politeness phenomena in Japanese", Journal of Pragmatics, 12, págs. 403-426.

McCarthy, M. (2000): "Mutually captive audiences: Small talk and the genre of close-contact service encounters". En Coupland, J. (ed.): Small Talk. London, Longman, págs. 1-25.

Meier, A. J. (1995): "Passages of politeness", Journal of Pragmatics, 24, págs. 381-392.

Moles, J. (1974): "Decisions and variability: The usage of address forms, pronouns and languages by Quechua-Spanish bilinguals in Peru", Anthropological Linguistics, 16, págs. 442-463.

Narbona, A., R. Cano, y R. Morillo (1998): El español hablado en Andalucía. Barcelona, Ariel.

Placencia, M. E. (1996): "Politeness in Ecuadorian Spanish", Multilingua, 15, págs. 13-34.

Placencia, M. E. (2004): "Rapport-building activities in corner shop interactions", Journal of Sociolinguistics, 8 , págs. 215-245.

Placencia, M. E. (en prensa): "Pragmatic variation in corner store interactions in Quito and Madrid". Sifianou, M. (1989): "On the telephone again! Differences in telephone behaviour: England vs. Greece", Language in Society, 18, págs. 527-544.

Sifianou, M. (1992): Politeness phenomena in England and Greece. A cross-cultural perspective. Oxford, Clarendon Press.

Spencer-Oatey, H. (2000): "Rapport management: A framework for analysis". En Spencer-Oatey, H. (ed.): Culturally Speaking Managing Rapport through Talk across Cultures. London, Continuum, págs. 11-45.

Spencer-Oatey, H. (2002): "Managing rapport in talk: Using rapport sensitive incidents to explore the motivational concerns underlying the management of relations", Journal of Pragmatics, 34, págs. 529-545.

Spencer-Oatey, H. (en prensa): "Motivations for politeness: Reconciling different perspectives through a rapport management approach".

Spencer-Oatey, H. y W. Jiang (2003): "Explaining cross-cultural pragmatic findings: Moving from politeness maxims to sociopragmatic interactional principles (SIPs)", Journal of Pragmatics, 35, págs. 1633-1650.

Tannen, D. (1994): Talking from 9 to 5: Women and Men in the Workplace. Language, Sex and Power. New York, Avon Books.

Thurén, B. M. (1988): Left Hand Left Behind. The Changing Gender System of a Barrio in Valencia, Spain. Stockholm Studies in Social Anthropology.

Traverso, V. (2001): "Syrian service encounters: A case of shifting strategies within verbal exchange", Pragmatics, 11, págs. 421-444.

Turnbull, W. (2003): Language in Action. Psychological Models of Conversation. Hove and New York, Psychology Press.

Valeiras Viso, J. M. (2002): "'Deja tu mensaje después de la señal': Despedidas y otros elementos de la sección de cierre en mensajes dejados en contestadores automáticos en Madrid y Londres".

En Placencia, M. E. y Bravo, D. (eds.): Actos de habla y cortesía en español. Munich, Lincom Europa, págs. 209-232.

Vázquez Orta, I. (1995): "A contrastive study of politeness phenomena in England and Spain". En Applied and Interdisciplinary Papers, Paper n. ${ }^{\circ}$ 267. Duisburg, Germany, L.A.U.D. 